\title{
ADAPTACIÓN TRANSCULTURAL DEL CUESTIONARIO KDQOL SF 36 PARA EVALUAR CALIDAD DE VIDA EN PACIENTES CON ENFERMEDAD RENAL CRÓNICA EN COLOMBIA
}

\author{
Karen Chaves, Andrés Duarte, Jasmín Vesga \\ Correspondencia: karen.chaves@boehringer-ingelheim.com \\ Recibido: Agosto 8 de 2013 Aceptado: Noviembre 4 de 2013 \\ Resumen
}

\begin{abstract}
El objetivo de este trabajo es adaptar transculturalmente, para el uso en Colombia, el cuestionario para calidad de vida en pacientes con enfermedad renal crónica terminal, KDQOL-SF-36 Kidney Disease Quality of Life (instrumento mixto). Al realizar esta adaptación, se busca que el significado y semántica de las preguntas sean consistentes para ser aplicados en población Colombiana. Método: para la realización de todo el proceso de adaptación se tomaron en cuenta las recomendaciones dadas por la Organización Europea para la Investigación y el Tratamiento del Cáncer (EORTC), la aplicación del cuestionario en los grupos pilotos se llevo a cabo mediante entrevista, autoadministración y entrevista en grupos focales. Resultados: durante el proceso de adaptación se realizaron diversos cambios en el cuestionario, la mayoría de ellos en la fase de traducción desde los lineamientos seguidos en la metodología. Conclusiones: en este momento en Colombia se cuenta con la versión adaptada de la escala KDQOL-SF-36, para pacientes con enfermedad renal crónica terminal.
\end{abstract}

Palabras clave: Enfermedad renal crónica terminal, calidad de vida, cuestionario, hemodiálisis, KDQOL-SF36.

\section{ADAPTATION TRANSCULTURAL KDQOL QUESTIONNAIRE SF 36 TO ASSESS QUALITY OF LIFE IN PATIENTS WITH CHRONIC KIDNEY DISEASE IN COLOMBIA}

\begin{abstract}
The aim of this work is to obtain a Colombian transcultural adaptation version of the instrument 'kidney disease quality of life' KDQOL - SF - 36 (mixed instrument) in subjects with end stage renal disease. By doing this work it is intended that the meaning and context of the questions be consistent to be applied in Colombian population. Method: For the accomplishment of the whole process of adjustment, the recommendations given by the European Organization for Research and Treatment of Cancer (EORTC) were taken in account; the application of the questionnaire in the pilot groups was carried out by interview, self administration and focal group interviews. Results: during the process of adjustment, several changes were made to the questionnaire, most of them in the translation phase from the standards followed in the methodology. Conclusions: Currently in Colombia the adapted version of the KDQOL-SF-36 scale is being used for subjects with end stage renal disease.
\end{abstract}

Key words: end-stage renal disease, quality of life, psychometric questionnaires, hemodialysis, $\mathrm{KD}$ QOL-SF36. 


\title{
ADAPTAÇÃO TRANSCULTURAL DO QUESTIONÁRIO KDQOL SF 36 PARA AVALIAR QUALIDADE DE VIDA EM PACIENTES COM DOENÇA RENAL CRÔNICA NA COLÔMBIA
}

\begin{abstract}
Resumo
O objetivo destetrabalho é adaptar transculturalmente para o uso na Colômbia o questionário para qualidade de vida em pacientes com doença renal crônica terminal KDQOL-SF-36 KidneyDiseaseQualityofLife (instrumento misto). Ao realizar esta adaptação procura-se que o significado e semântica das perguntas sejam consistentes para ser aplicados em população Colombiana. Método: para a realização de todo o processo de adaptação se tiveram em conta as recomendações dadas pela Organização Europeia para a Pesquisae o Tratamento do Câncer (EORTC), a aplicação do questionário nos grupos pilotos, se levou a cabo mediante entrevista, autoadministração e entrevista em grupos focais. Resultados: durante o processo de adaptação se realizaram diversos câmbios ao questionário a maioria de eles na fase de tradução desde os lineamentos seguidos na metodologia. Conclusões: em este momento na Colômbia se conta com a versão adaptada da escala KDQOL-SF-36 para pacientes com doença renal crônica terminal.
\end{abstract}

Palavras chave: Doença renal crônica terminal, qualidade de vida, questionário, hemodiálise, KDQOL-SF36.

\section{Introducción}

La enfermedad renal crónica es un problema de salud pública a nivel mundial, que implica la pérdida progresiva de la función renal, la prevalencia de dicha enfermedad ha ido en aumento un $6.8 \%$ anual, la incidencia ha aumentado desde 33,3 pacientes por cada millón de habitantes en 1993 a 167,8 pacientes por cada millón de habitantes en 2005 en Latinoamérica (1).

En Latinoamérica la frecuencia de enfermedad renal es similar en todos los países de la región. Para Colombia se ha estimado en el orden de 12 por 100.000 habitantes para una población de 43.000 .000 habitantes (2) La mortalidad por enfermedad renal es de 3,7 por 100.000 personas entre los 15 y 60 años. (3).

Los pacientes con enfermedad renal crónica deben someterse a tratamientos no curativos, altamente invasivos, demandantes y que involucran altos costos para el paciente y su familia, a nivel físico, psicológico, social y económico, los cuales deben acompañarse de una dieta estricta, toma de medicamentos y restricción de líquidos (4), lo cual genera un deterioro considerable de la calidad de vida.

La percepción de calidad de vida relacionada con salud, se expresa en términos de bienestar, como un proceso dinámico y cambiante (5), que varía según el sistema de valores de los individuos (6), movido por esto y muchas otras razones, la calidad de vida a nivel mundial se ha convertido en un indicador importante en los servicios de salud.

En las últimas décadas se han desarrollado diferentes instrumentos para medir la calidad de vida, tanto en la población general, como en grupos específicos de pacientes. Los cuestionarios de calidad de vida de acuerdo con su aplicación y foco son clasificados en: genéricos, específicos y mixtos o modulares.

Un instrumento genérico es aplicable ampliamente a una población y permite comparaciones de diferentes grupos de pacientes, pero no focaliza adecuadamente sobre un área de interés o problema clínico en particular. Los instrumentos específicos focalizan sobre problemas asociados con una enfermedad determinada o grupos de pacientes, no permite comparaciones entre condiciones diferentes por tanto su aplicabilidad puede ser limitada. Un instrumento mixto o modular combina las características tanto del genérico, como del específico. (7)

KDQOL Kidney Disease Quality of Life Instrument fue desarrollado para los pacientes en hemodiálisis y diálisis peritoneal, e incluye el SF-36 como núcleo genérico. 
El cuestionario contiene 43 ítems específicos para pacientes con enfermedad renal crónica distribuidos de la siguiente forma entre 11 dimensiones específicas para la enfermedad: síntomas/problemas (12 ítems), efectos de la enfermedad renal en la vida diaria (8 ítems), carga de la enfermedad (4 ítems), situación laboral (2 ítems), función cognitiva (3 ítems), relaciones sociales ( 3 ítems), función sexual (2 ítems), sueño (4 ítems), apoyo social (2 ítems), actitud del personal de diálisis (2 ítems), y satisfacción del paciente (1 ítem).

También incorpora los 36 ítems del SF-36 que se distribuyen en 8 dimensiones de salud física y mental: función física ( 10 ítems), limitaciones de rol por problemas de salud físicos ( 4 ítems), limitaciones de rol por problemas de salud emocionales (3 ítems), la función social (2 ítems), bienestar psicológico (5 ítems), dolor (2 ítems), vitalidad/cansancio (4 ítems), y percepción global de la salud (5 ítems).

En el último ítem del KDQOL-SF 36 los pacientes tienen la posibilidad de evaluar su salud en general en una escala de 0-10, donde 0 equivale a "peor salud posible (tan malo o peor que estar muerto)" y 10 equivale a "la mejor salud posible".

Este es uno de los instrumentos más utilizado internacionalmente en estudios multicéntricos $(8,9,10,11,12)$ que cuenta con valores adecuados de confiabilidad y validez, para población con enfermedad renal crónica, además es un instrumento mixto, lo cual favorece realización de comparaciones a nivel específico de la enfermedad renal crónica, diferencias de tratamientos y con población general, es uno de los instrumentos que tiene más publicaciones de adaptación y validación a diversos idiomas en el mundo $(13,14,15,16,17,18)$.

En Colombia no existe un instrumento validado para evaluar calidad de vida en pacientes con enfermedad renal crónica, y dadas las características previamente descritas del cuestionario KDQOL SF 36, se dio inicio al proceso de validación del mismo, donde la adaptación transcultural es el primer paso a realizar.

\section{Metodología}

\section{Adaptación transcultural}

Para el proceso de adaptación transcultural se adoptaron las recomendaciones del grupo EORTC, el cual propone el siguiente proceso:

\section{Traducciones al español hablado en Colombia:}

El cuestionario KDQOL SF 36 en la versión española de España, fue traducido por dos traductores de manera independiente quienes tenían como lengua madre el español colombiano, el primer traductor médico internista colombiano con amplia experiencia en manejo de pacientes crónicos, la segunda persona encargada de la traducción es una médica colombiana, internista, especialista en epidemiología clínica, de cada uno de ellos se obtuvo una versión realizada de manera independiente, con los cambios que cada uno consideró sin uso de lenguaje técnico, necesario para el adecuado entendimiento y aplicación del cuestionario por la población colombiana.

\section{Comparación de traducciones}

Con la dirección de uno de los autores del presente estudio, se discutió con los traductores cada uno de los items de la escala, evaluando la semántica y facilidad de entendimiento, sin cambiar el sentido original del cuestionario, en los ítems donde existió concordancia entre los cambios sugeridos por los dos traductores se dejó la modificación de esta forma, cuando no hubo concordancia entre los traductores, se discutió con ellos para llegar a un acuerdo lo cual sucedió en todos los casos, se obtuvo de esta forma la versión preliminar del instrumento.

\section{Traducción inversa del español colombiano a inglés}

Un traductor con dominio de las dos lenguas (español e inglés), realizó la traducción inversa a inglés, de los ítems que fueron modificados en la versión preliminar, esta versión fue comparada con la versión original de la escala en idioma inglés, buscando que existiera concordancia semántica entre las traducciones, donde no había concordancia era necesario modificar la versión del español colombiano.

\section{Prueba piloto}

La versión preliminar fue llevada a prueba piloto, para esta fase se pidió la colaboración de (40) pacientes con enfermedad renal crónica terminal, elegidos por conveniencia, en dos unidades renales en Bogotá, Colombia. En la prueba se administró el cuestionario en entrevista individual o de forma auto- administrada, posteriormente mediante entrevista por grupo focal conducida, revisando cada ítem del cuestionario con los participantes en el estudio, se evaluó, la dificultad para responder las preguntas, confusión generada por la pregunta, dificultad para entender la pregunta y 
molestia generada por la pregunta, cuando se encontró alguna dificultad se solicitó a los pacientes un refraseo que se recolectó en el mismo formato de evaluación.

\section{Elaboración de la versión final del instrumento}

Teniendo en cuenta todos los cambios y el proceso descrito previamente, se realizó la versión del cuestionario KDQOL SF 36 adaptado transculturalmente al español de Colombia, para pacientes con enfermedad renal crónica en terapias de reemplazo renal.

\section{Resultados}

Proceso de traducción al español hablado en Colombia y comparación de traducciones

En las instrucciones del cuestionario los dos traductores coincidieron en cambiar en el punto 1 "Las preguntas que siguen refieren a lo que usted piensa sobre su salud", por "Las preguntas que siguen hacen referencia a lo que usted piensa sobre su salud." En el punto 3 existió acuerdo y se cambió "Por favor, conteste cada pregunta rodeando con un círculo el número correspondiente o rellenando la respuesta tal como se indique." por "Por favor, conteste cada pregunta marcando con un círculo o una x el número correspondiente o llenando la respuesta tal como se indique." En la pregunta 1 y 2 los dos traductores coincidieron en que no era necesario realizar cambios de la versión española neutra.

En la pregunta 3, item $b$ los dos traductores sugirieron el cambio de "Esfuerzos moderados, como mover una mesa, pasar la aspiradora, jugar a los bolos o caminar más de 1 hora" por "Esfuerzos moderados, como mover una mesa, pasar la aspiradora, o caminar más de 1 hora". Los dos traductores resaltaban la necesidad de retirar "jugar bolos" del ítem, dado que en Colombia es un deporte practicado de manera esporádica, no difundido de manera generalizada en la población de nuestro país y esto podría dificultar el entendimiento de este punto. Se revisó el cuestionario en su versión original, donde se menciona en el ítem a "jugar golf" deporte difundido en menor medida que los bolos en Colombia, por esta razón se tomó la decisión de suprimir este aspecto en el ítem.

En la pregunta 3, ítem $h$ e i los dos traductores coinciden en cambiar "manzanas" por "cuadras". En la pregunta 4, ítem a $c d$ y pregunta 5 , ítem a y c los dos traductores coincidieron en cambiar "actividades cotidianas" por "actividades diarias". En la pregunta 6 a 8: No se realizaron modificaciones.
En la pregunta 9 se realizó el cambio en el encabezado de la pregunta, sugerido por el traductor 1 y aceptado por el traductor 2: "Las preguntas que siguen se refieren a cómo se ha sentido y cómo le han ido las cosas durante las 4 últimas semanas. En cada pregunta responda lo que se parezca más a cómo se ha sentido usted." por "Las preguntas que siguen hacen referencia a cómo se ha sentido durante las 4 últimas semanas. En cada pregunta responda lo que se parezca más a cómo usted se ha sentido." En las preguntas 10-12: no se realizan modificaciones.

En la pregunta 13, sugerido por el traductor 1 y aceptado por el traductor 2 se realiza el cambio en el encabezado de la pregunta: "Las preguntas que siguen se refieren a cómo se ha sentido y cómo le han ido las cosas durante las 4 últimas semanas. Dé a cada pregunta la respuesta que se parezca más a cómo se ha sentido usted." Por "Las preguntas que siguen se refieren a cómo se ha sentido durante las 4 últimas semanas. Marque en cada pregunta lo que se relacione más a cómo se ha sentido usted."

En la Pregunta 15, ítem e el traductor 2 sugiere cambio de "personal sanitario" a "personal de salud", aceptado por el traductor 1. En la pregunta 15, ítem $f$ los dos traductores sugirieron el cambio de "tensión nerviosa" por "nerviosismo". En la pregunta 16, sugerido por traductor 2 aceptado por traductor 1 se cambia "iHasta qué punto supusieron un problema cada una de las siguientes cosas, durante las 4 últimas semanas?" por "¿Hasta qué punto fueron un problema cada una de las siguientes cosas, durante las 4 últimas semanas?" Pregunta 17 a 24: no se realizaron modificaciones. Se mantuvo la estructura y esquema del instrumento, las palabras que son resaltadas y orden de las preguntas.

\section{Traducción inversa del español colombiano a inglés}

No hubo evidencia de cambios significativos en la estructura y semántica de los cambios realizados de acuerdo con el cuestionario en idioma inglés, por lo tanto, se mantuvieron los cambios realizados en la comparación de las traducciones.

\section{Prueba Piloto}

En la prueba piloto realizada, se encontraron las siguientes características en la muestra: grupo conformado por 12 mujeres y 18 hombres, la edad tuvo una media de 48,7 años (ds: 15,1 ), los valores estuvieron entre 21 y 71 años, la mayoría de los participantes tenían estudios: 
bachillerato completo seguido por primaria completo, y universitario completo y estado civil casado, seguido en orden de frecuencia por soltero.

Tabla 1. Distribución de los pacientes de la prueba piloto por escolaridad.

\begin{tabular}{|l|c|c|}
\hline \multicolumn{1}{|c|}{ Escolaridad } & n & \% \\
\hline Primaria completa & 5 & 16,6 \\
\hline Bachillerato completo & 12 & 40 \\
\hline Bachillerato incompleto & 1 & 3,3 \\
\hline Técnico & 4 & 13,3 \\
\hline Universitario completo & 5 & 16,6 \\
\hline Universitario incompleto & 3 & 10 \\
\hline Total & 30 & 100 \\
\hline
\end{tabular}

Tabla 2. Distribución de los pacientes de la prueba piloto por estado civil.

\begin{tabular}{|l|c|c|}
\hline \multicolumn{1}{|c|}{ Estado civil } & n & \% \\
\hline Soltero & 9 & 30 \\
\hline Unión libre & 1 & 3,3 \\
\hline Casado & 18 & 60 \\
\hline Divorciado & 1 & 3,3 \\
\hline Viudo & 1 & 3,3 \\
\hline Total & 30 & 100 \\
\hline
\end{tabular}

El tiempo promedio requerido para diligenciar el cuestionario fue de 29 minutos, con un rango de tiempo de 15 a 55 minutos, lo cual encontramos en relación con el grado de escolaridad que tenía el participante con tiempos mayores de respuesta, en personas con grado de escolaridad más bajo.

Teniendo en cuenta las características enunciadas anteriormente, se consideró que la muestra del grupo piloto era representativa de los pacientes para los cuales el cuestionario estaba diseñado, en términos sociodemográficos.

En el título de las instrucciones del instrumento, varios pacientes sugirieron cambiar "instrucciones para rellenar el cuestionario" por "instrucciones para completar el cuestionario". Así mismo se sugirió el cambio de la palabra "rellenar" por "completar" al final de las instrucciones.

En la pregunta 14, ítem $d$ varios pacientes sugirieron el cambio de la palabra "picores" por "rasquiña". Solo un paciente sugirió cambio en la escala visual de la pregunta 17, para posicionar de manera vertical, cambio que no compete en el proceso de adaptación transcultural y cambio sugerido solo por uno de los participantes de la prueba piloto.

Durante la prueba piloto varios participantes sugirieron el cambio en la pregunta 14, ítem e de "sequedad en la piel" por "resequedad en la piel" sin embargo según el Diccionario de la Real Academia Española la palabra "resequedad" no existe (19), motivo por el cual, el cambio no fue realizado.

Las escalas de calificación fueron adoptadas adecuadamente y no se refirió mayor dificultad para el adecuado diligenciamiento del cuestionario.

\section{Discusión}

El proceso de traducción y adaptación transcultural ha sido considerado esencial para comparaciones entre estudios de diferentes países, lenguajes y culturas; una traducción lingüísticamente adecuada no es suficiente ya que algunos ítems deben ser adaptados culturalmente para preservar el significado conceptual del cuestionario. La adaptación transcultural es el paso inicial del proceso de validación, el cual se requiere cuando existen versiones del instrumento en el mismo idioma pero desarrolladas en culturas diferentes. (20)

Ya que el disponer de instrumentos adaptados y validados, abre posibilidades diversas como comparaciones entre diferentes países y lenguajes, cada vez se hace más necesario contar con este tipo de instrumentos. En Colombia no hay disponibilidad de ningún instrumento que cuente con estas características, para la evaluación de calidad de vida en pacientes con enfermedad renal crónica.

Este trabajo presenta el proceso de adaptación transcultural de un cuestionario para medir calidad de vida en pacientes con enfermedad renal crónica en terapia de reemplazo renal, patología con un aumento creciente en nuestra población, y con tratamientos que impactan a múltiples niveles el bienestar de la persona. Como resultado se cuenta con la versión del cuestionario KDQOL SF 36 para el español de Colombia.

Este proceso fue desarrollado de manera sistemática, y estandarizada de acuerdo con las recomendaciones del grupo EORTC, para obtener esta versión se realizaron algunas modificaciones a la versión del español neutro, 
que no conllevan cambios semánticos ni contextuales del instrumento en su versión original, y de manera adicional permite una fácil y adecuada interpretación en nuestra población.

Se podría considerar cuestionable el tamaño de muestra utilizado cuarenta (40) participantes, pero cabe resaltar que el tamaño fue mayor al utilizado en la mayoría de las recomendaciones de adaptación transcultural en diferentes protocolos.

Una de las limitaciones para la aplicación de este estudio, es la escasez de publicaciones en español especialmente a nivel Latino América, que adapten y validen el cuestionario KDQOLSF 36, para la realización de estudios de comparación en dichas regiones $\operatorname{Sin}$ embargo, conocemos por el momento la adaptación realizada en Argentina y en Chile, así mismo, dado el gran uso a nivel mundial de este cuestionario, este estudio espera ser una invitación y punto de partida para la realización de estudios de esta índole en otros países de Latino América.

Actualmente se está realizando el estudio de validación del cuestionario KDQOL SF 36, lo cual es necesario para determinar las propiedades psicométricas del instrumento y su posterior aplicación en la práctica clínica e investigación, con la realización de estudios a diferente escala a nivel nacional e internacional.

\section{Conclusión}

La escala KDQOL SF 36 se encuentra adaptada al español para Colombia. Se considera necesario continuar con el proceso de validación de la misma para su posterior utilización en diferentes estudios.

\section{Referencias}

1. Cusumano A, González M. Chronic kidney diseases in latin america: Time to improve screening and detection. Clin J Am Soc Nephrol. 2008; 3: 594-600.

2. Challu A, Burgos R, Santos R, Feler D. La nefrología en latinoamerica. Estado actual pautas para su desarrollo. 1999.

3. Lopez C, Jaramillo A, Jaimes J. Prevalencia de enfermedad renal y entidades asociadas: una base para la promoción de la salud en la población de Simijaca. Revista de la facultad de medicina. 2005; 10: 63-70

4. Contreras F, Esguerra G, Espinosa JC, Gutierrez C, Fajardo L. Calidad de vida y adhesión al tratamiento en pacientes con insuficiencia renal crónica en tratamiento de hemodialisis. Univ Psychol Bogotá. 2006; 5: 487-499.
5. Porter G. Assessing the outcome of rehabilitation in patients with end-stage renal disease. Am J Kidney Dis.1994;24:22-27.

6. Gonzalez V, Lobo N. Calidad de vida en los pacientes con insuficiencia renal crónica terminal en tratamiento sustitutivo de hemodiálisis. Aproximación a un proyecto integral de apoyo. Revista de la sociedad Española de enfermeria nefrologica.2001; 4: 6-12.

7. Cagney K, Wu Albert et al. Formal literature review of quality of life instruments used in end stage renal disease. Am J Kidney Dis. 2000; 36 : 327-336.

8. Mapes DL, Lopez AA, Satayathum S, McCullough KP, Goodkin DA, Locatelli F, et al. Health- related quality of life as a predictor of mortality and hospitalization: The dialysis outcomes and practices patterns study (DOPPS). 2003; 137: 339-349.

9. Fukuhara S, Lopes AA, Bragg JL, Kurokawa K, Mapes DL, Akizawa $\mathrm{T}$, et al. Health related quality of life among dialysis patients on three continent: The dialysis outcomes and practice patterns study. Kidney international. 2003; 64: 1913-1910.

10. Gayle F, Soyibo AK, Gilbert DT, Manzanares J, Barton EN. Quality of life in end stage renal disease: a multicenter comparative study. West Indian Medicine.2009;58: 235

11. Unruh ML, Weisbord SD, Kimmel PL. Health related quality of life in nefrology research and clinical practice 2005. Seminars in Dialysis. 2005; 18: 82-90.

12. Edgell ET, Coons SJ, Carter WB, Kallich JD, Mapes D, Damush $\mathrm{TM}$, et al. A review of health related quality of life measures used in end stage renal disease. Clinical therapeutics. 1996; 18: 887-938.

13. Hays RD, Kallich JD, Mapes DS, Coons SJ, Carter WB. Development of the kidney disease quality of life (KDQOL) instrument. Qual Life Res. 1994; 3: 329-338.

14. Garcia F, Lopez K, De Alvaro F, Alvarez U, Alonso J. Salud percibida en pacientes que comienzan tratamiento renal sustitutivo, validación preliminar de la versión española del KDQOL SF. Nefrología. 1998; 18: 66.

15. Hays RD, Amin N, Apolone G, Kamberg C, Kallich J, Coons S, et al. Kidney disease quality of life short form (KDQOL SF36). Version SF 36.: a manual for use an scoring (Italian questionary, Italy). Santa Mónica, CA: RAND; 1997.

16. Hays RD, Amin N, Bullinger M, Mapes D, Kamberg C, Kallich J, et al. Kidney disease quality of life short form (KDQOL SF36). Version 1.2: a manual for use an scoring (German questionary, Germany). Santa Mónica, CA: RAND; 1997.

17. Hays RD, Amin N, Leplege A, Carter WB, Mapes DL, Kamberg $\mathrm{CJ}$, et al. Kidney disease quality of life short form (KDQOL SF36). Version 1.2: a manual for use an scoring (French questionary, France). Santa Mónica, CA: RAND; 1997.

18. Hays RD, Amin N, Fukahara S, Coons S, Carter WB, Mapes DL. Kidney disease quality of life short form (KDQOL SF36) Version 1.2: a manual for use an scoring (Japanese questionary, Japan). Santa Mónica, CA: RAND; 1997.

19. Diccionario de la Real Academia de la Lengua Española. [sede Web]. Madrid: Real Academia de la Lengua Española. Disponible en: htto://www.rae.es/rae.html.

20. Calvo O, Oliveros R, Sánchez R. Adaptación transcultural del formulario EORTC QLQ CR-29 para su aplicación en pacientes con cáncer de recto en el Instituto Nacional de Cancerología de Colombia. Rev Colomb Cancerol. 2010; 14:189-198. 